Proceedings of the ASME 2015 34th International Conference on Ocean, Offshore and Arctic Engineering

May 31-June 5, 2015, St. John's, Newfoundland, Canada

OMAE2015-41529

\title{
OPTIMISING NUMERICAL WAVE TANK TESTS FOR THE PARAMETRIC IDENTIFICATION OF WAVE ENERGY DEVICE MODELS
}

\author{
John V. Ringwood* \\ Centre for Ocean Energy Research \\ Maynooth University, Ireland \\ john.ringwood@eeng.nuim.ie
}

\author{
Josh Davidson \\ Centre for Ocean Energy Research \\ Maynooth University, Ireland \\ josh.davidson@eeng.nuim.ie
}

\author{
Simone Giorgi \\ Centre for Ocean Energy Research \\ Maynooth University, Ireland \\ simone.giorgi@eeng.nuim.ie
}

\begin{abstract}
While linear and nonlinear system identification is a well established field in the control system sciences, it is rarely used in wave energy applications. System identification allows the dynamics of the system to be quantified from measurements of the system inputs and outputs, without significant recourse to first principles modelling. One significant obstacle in using system identification for wave energy devices is the difficulty in accurately quantifying the exact incident wave excitation, in both open ocean and wave tank scenarios. However, the use of numerical wave tanks (NWTs) allow all system variables to be accurately quantified and present some novel system tests not normally available for experimental devices. Considered from a system identification perspective, this paper examines the range of tests available in a NWT from which linear and nonlinear $d y-$ namic models can be derived. Recommendations are given as to the optimal configuration of such system identification tests.
\end{abstract}

\section{Introduction}

System identification, which can be considered to be the dynamical equivalent of static function approximation, has its origins in the methods developed by Legendre, Laplace and Gauss in the 19th century, which were used to fit functional forms to (typically) astronomical data [1]. However, since the 1960's, the field of dynamical system identification has been consolidated and many successful applications have been reported using a wide variety of methods across a broad range of application

*Address all correspondence to this author. areas. [2] contains a succinct synopsis of modern system identification, while [3] provides a more comprehensive treatment. System identification finds particular application in areas where:

- First principles models are too difficult to formulate, or

- The dynamics of a system change with time and on-line model updating is required (e.g. in adaptive control).

In many cases, system identification is seen as a pragmatic and time-efficient method of obtaining dynamical models in situation where system variables (inputs, outputs) are relatively straightforward to measure. In addition, 'grey-box' methods allow the use of system identification methods to determine the parameters of system models, where the parametric structure is determined from first principles, with various shades of 'grey' being used to denote the combination of first principles and data-based techniques [2].

In wave energy modelling, system identification is generally only used for finding suitable low-order approximations to nonparametric impulse response [4] or frequency response data [5] from boundary-element solvers. However, such use relates more to model order reduction (in the control systems vernacular) as opposed to model building. One particular difficulty in applying system identification techniques to wave energy devices is the difficulty in making accurate measures of the wave excitation. This is true even in wave tanks, where unwanted wave reflections can contaminate the generated excitation signal. While system identifications can be shown to be robust to uncorrelated noise they are, in general not robust to highly correlated disturbances. 
Yet, despite these difficulties, there is considerable motivation to apply system identification techniques to wave energy device modelling. With some minor exceptions, boundary element methods (BEMs) generally are used to develop linear hydrodynamic models, while more computationally demanding methods such as computational fluid dynamics (CFD) or smooth particle hydrodynamics (SPH) can be used to obtain more accurate (nonlinear) simulations. However, in both cases, recourse is made to numerical methods and while some structural model information is preserved using BEMs, there is little transparency between the physical system and the models in either the BEM or CFD/SPH case. Therefore, the move to use a data-based approach to determine (computationally compact) linear or nonlinear parametric models using system identification is appealing.

Recently, NWTs have been proposed to simulate the behaviour of wave energy devices under very controlled conditions $[6,7]$. NWTs have the capability to accurately simulate the motion of wave energy devices under a range of situations and for a range of excitations. In addition, and importantly, NWTs also permit the measurement and analysis of many intermediate variables (such as individual forces) which can allow the isolation of various model components for modelling. However, one drawback of NWTs is the significant computational time associated with the CFD calculations.

One important issue in system identification is the specification of the system input signal. In many applications, it is not possible to independently specify the input signal, where the input signal is dependent on other signals (e.g. feedback or error signals), for example in the case of adaptive control [8]. Where the input is free to choose, the problem reduces to one of experiment design [9]. In general, a good test signal should satisfy the following properties:

- Good coverage of the frequencies where the system has a significant frequency response,

- Good coverage of the full input and output signal ranges (if the system is nonlinear), and

- Economical use of the test time.

This paper examines the issue of experiment design for the identification of wave energy device models using NWT experiments. We utilise an artificial neural network (ANN) model for the target parametric model structure and demonstrate the validity of the identified models on independent test data. The remainder of the paper is organised as follows: Section 3 details the important issues in system identification in general, while Section 2 examines the salient aspects of a NWT relevant to system identification tests. Then, a case study is outlined in Section 4 and a series of experiments for this system, using a NWT, are documented in Section 5. The identification of dynamical ANN models is carried out in Section 6 and these models are validated in Section 7. Finally, conclusions are drawn in Section 9.

\section{Numerical wave tanks}

NWTs are computer implementations, in either 2 or 3 dimensions, of wave tanks that normally have some wave generation facility and can disperse waves at the boundaries to avoid reflection [10]. NWTs can be implemented using BEMs or CFD. CFD-based NWTs offer fully nonlinear hydrodynamic calculations and solve the Navier-Stokes equations [11] which govern the transfer of mass, momentum and heat, by discretizing the domains of space and time to form a system of linear algebraic equations, which are computer implementable. NWTs provide a virtual cost-effective alternative to real wave tanks and, in some respects, have advantages over real wave tanks inculding:

- More cost-effective evaluation of device motion and power production capabilities

- Greater control over the forces, including wave motion, applied to the test device

- Constrain the device motion on any desired degrees of freedom

- Resolution of some individual hydrodynamic forces, including restoring forces and viscous forces

- Test at full scale

In wave energy research, NWTs are normally used to assess WEC behaviour, usually to get an early indication of the power conversion capabilities of a device. The types of responses that can be achieved using NWTs include:

(a) Free response tests, which activate the fundamental system dynamics,

(b) Forced response tests, where responses combine the steadystate response to forced inputs and the transient response due to the fundamental system dynamics.

For (b) above, excitation can be applied via either input waves, where there is a limitation in the spectral envelope of the excitation signal, or via direct manipulation of the device itself (e.g. via a power take-off (PTO) system), where there are no limitations on the spectral envelope of the input signal.

However, NWTs are not without their drawbacks. The chief disadvantage, over a conventional wave tank, is the excessively long time to perform the numerical computation of the response, and typical computation times can be up to 1000 times the simulation time i.e. $1 \mathrm{~s}$ of simulation time takes $1000 \mathrm{~s}$ to compute. In addition, NWTs can take considerable experience to use well, in particular setting up a spatial mesh which offers a reasonable compromise between computation time and accuracy.

This paper directly addresses the computational complexity issue by using NWTs to develop sets of representative responses which can subsequently be used to produce computationally efficient parametric models. The construction of these system identification tests needs to be carefully considered, so that representative input/output behaviour over the operational space is recorded, while minimising overall computation time. 


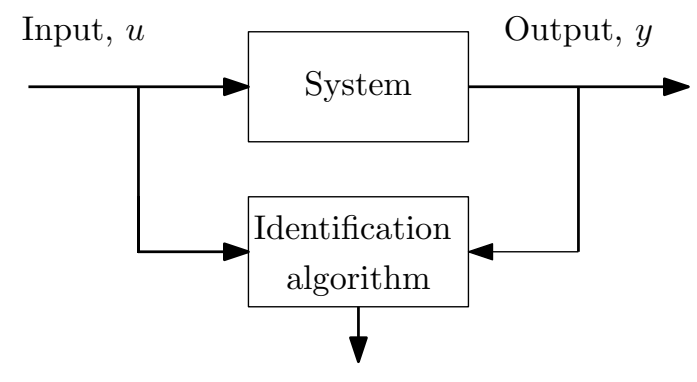

Parameters, $\Theta$

FIGURE 1. System identification principle

\section{System Identification}

The fundamental principle of system identification is captured in Fig.1. The identification procedure follows the following steps:

1. A parametric structure is chosen for the model,

2. A suitable input signal, $u$ is synthesised and input to the system,

3. The input signal, $u$ and resulting output signal, $y$ are recorded

4. An identification algorithm is used to determine the optimal parameter vector, $\Theta$, which minimises some error metric between the actual measured output $y$ and that produced by the identified parametric model.

Normally, discrete time models of the form:

$$
y_{k}=M\left(\Theta, u_{k}, y_{k}\right)
$$

are identified from samples input and output data where, in general, $M\left(\Theta, u_{k}, y_{k}\right)$ is a difference operator, and $M$ can be linear or nonlinear. However, identification of continuous-time models can also be performed [12].

\subsection{Model parametric structures}

Choice of the model parametric structure is key if a representative model is to be determined. The model structure may be inspired from physical system knowledge and considerations (white-box modelling) or completely based on the recorded data itself (black-box modelling). There are also many possibilities within these two extremes which are denoted by shades of grey e.g. off white, slate grey, smoke grey, etc. [2]. In terms of some physical information for the identification of hydrodynamic models, we might generally include a term related to acceleration (mass, added mass), velocity (radiation damping) and position (restoring force). If, for example, if viscous damping is likely to feature significantly (depending on the WEC shape and likely velocity range), we could include a term proportional to the square of velocity, etc.
In general, whether the model structure is inspired by physical phenomena or purely derived from the data, we need to determine a parsimonious structure for the model which will work well with the identification data, but also generalise well to other data. A compatible requirement is that we don't want to add complexity to the model for little gain.

If the model structure is deemed to be linear then, for the single input, single output case we get an AutoRegressive with eXogenous input (ARX) model of the form:

$$
y_{k}=a_{1} y_{k-1}+\ldots+a_{n} y_{k-n}+b_{0} u_{k}+\ldots+b_{m} u_{k-m}
$$

In this linear case, the structure determination problem reduces to the specification of $n$ and $m$. However, if we deem that a nonlinear structure is necessary, then the form, and complexity level, of the nonlinearity must be chosen. One possibility is to choose a polynomial nonlinearity, which results in the following Volterratype model:

$$
\begin{array}{rr}
y_{k}= & a_{11} y_{k-1}+\ldots+a_{n 1} y_{k-n}+b_{01} u_{k}+\ldots+b_{m 1} u_{k-m} \\
+ & a_{12} y_{k-1}^{2}+\ldots+a_{n 2} y_{k-n}^{2}+b_{02} u_{k}^{2}+\ldots+b_{m 2} u_{k-m}^{2} \\
+ & c_{10} y_{k-1} u_{k}+\ldots+c_{n 0} y_{k-n} u_{k} \\
+ & \ldots
\end{array}
$$

In the structure of (3), we also need to specify the maximum polynomial orders for the terms involving $u$ and $y$ alone, as well as the cross-product terms. Another alternative general nonlinear parametric structure can be specified using ANNs, in either multi-layer perceptron (MLP) or radial-basis function (RBF) form. The basis functions in MLPs provide global support, while those employed in RBFs provide local support. In the case of MLPs, the nonlinear structure is specified by the number of layers of nonlinear neurons and the number of neurons in each layer. For the RBF case, a signle layer of nonlinear neurons is always employed, so the nonlinear complexity is specified by the number of basis functions employed.

Depending on the availability of intermediate measurements, system models can be decomposed into subsections, which can be individually identified. For example, Fig. 2 shows a decomposition of a WEC model so that individual models for the main dynamics and the excitation force kernel can be identified, providing a measurement of the excitation force, $F_{e}$ is available.

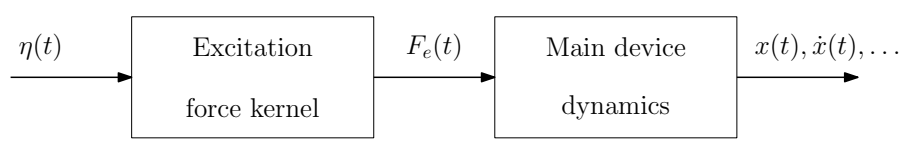

FIGURE 2. WEC model decomposition 


\subsection{Input signal synthesis}

For linear systems, the requirement on the input signal is simple: make sure that the input signal covers the frequency range of interest. For the wave energy case, the frequency range could be ascertained from either; some rough measurement of the resonant frequency and bandwidth of the device, or the range of sea frequencies in which the WEC is expected to operate. Usually these two frequency ranges are well connected.

Typically useful signals for the identification of linear systems are pseudo-random binary sequences, which have a flat frequency spectrum, or multi-sinusoids which contain a set of closely-packed frequencies. For nonlinear systems, there is an additional input/output signal amplitude dependence in the model, so the full operational range of amplitudes will also, ideally, need to be present in the identification signal. Therefore pseudo-random sequences with randomly varying amplitudes (or random amplitude, random period [RARP]) can be employed for nonlinear systems, or chirp and multi-sine signals are also useful, since the input signal varies continuously over the allowable input amplitude range [13].

Fig. 3 shows an example of a chirp, RARP and multisine signal. The spectral content and amplitude distributions of these signals are then plotted in Fig.4, displaying their respective frequency and amplitude domain coverage. Each signal has different attributes regarding their spectral and amplitude properties, and also regarding the dependence of these properties on the signal length. Obviously from a CFD computation standpoint - the shorter the simulation length the better.

The chirp signal's frequency linearly sweeps a desired range during the signal, therefore its frequency content is distributed fairly evenly across that range. The amplitude of the chirp signal is well bounded, with free choice in setting the maximum amplitude. However there is no choice over the amplitude distribution which is well defined and has two peaks near the extremes where the signal slows, stops and changes direction every oscillation.

The frequency content and amplitude distribution of the RARP vary due to the inherent randomness of the signal. However as the signal length increases, the amplitude distribution converges to an even coverage of the desired range and the frequency content converges to a distribution influenced by the maximum allowable random switching period (1s for the signal in Fig.3). The amplitude distribution can be controlled by filtering the random numbers produced for the amplitude with a desired probability distribution function (e.g. Gaussian).

The multisine allows strong control over the spectral content with free choice for the amplitude of each harmonic. The amplitude distribution is determined by the amplitude and the phases of the harmonic components, which leads to control over the amplitude content in the mutisine signals through phase optimisation techniques [13]. The multisine signal in Fig.3 is designed with a fundamental frequency of $0.05 \mathrm{~Hz}$ and equal amplitude for every harmonic up to $1 \mathrm{~Hz}$ with randomly assigned phases.
Free surface elevation and wave excitation force signals are constrained by underlying fluid dynamics (e.g. no square waves), therefore the possibility of utilising RARP or chirp signals is limited to excitation using an external (e.g. PTO) force input.

\subsection{Recording of input/output signals}

In wave tanks, (usually optical) motion capture systems are used to determine the device motion and wave gauges are used to measure the actual free surface elevation excursions which impact the device. In a NWT, motion and free surface elevation variations are easily measurable, but several additional measurement may also be made. For example, individual forces on the WEC (e.g. excitation force, viscous forces, etc) may be resolved, which may help in identifying subsections of the WEC model.

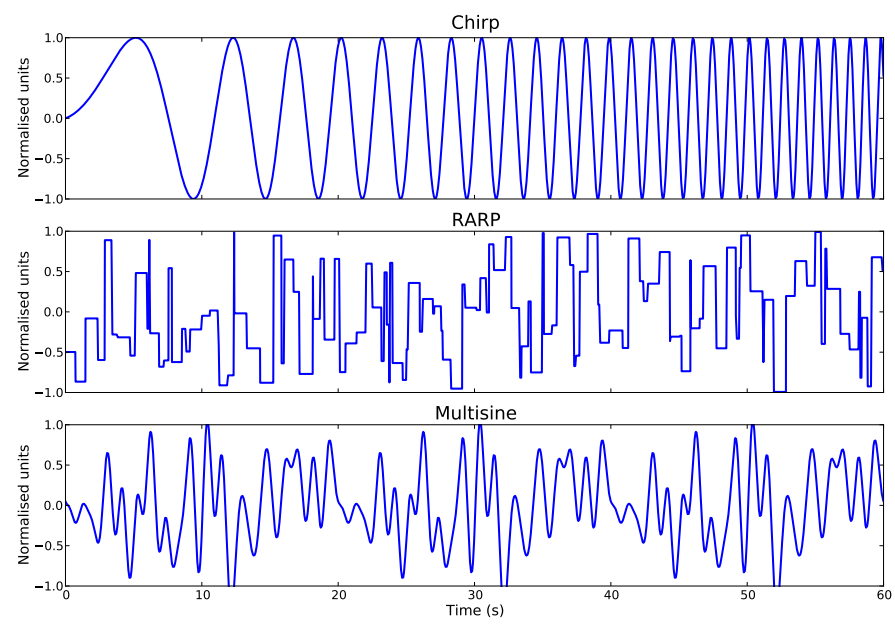

FIGURE 3. Time series of potential identification signals
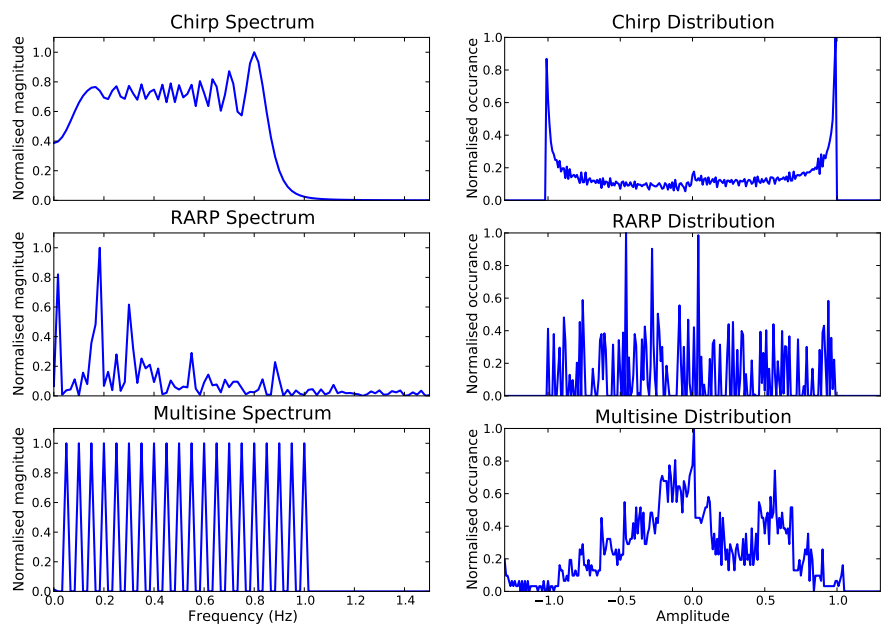

FIGURE 4. Spectrum and amplitude distribution for signals in Fig.3 


\subsection{Parameter determination}

If the model structure is linear and the error criterion employed is the quadratic output error (sum of squares), the identification problem can be formulated as a set of linear equations and solved using linear least squares. In all other situations, iterative techniques are required, usually relying on numerical optimisation routines. In particular, for nonlinear model structures, the performance surface is usually multi-modal (many local minima) requiring care in the use of line search algorithms. In such cases, the ability to provide good initial parameter values (from $a$ priori information) can be particularly beneficial. Alternatively, concurrent search algorithms, such as genetic algorithms or particle swarm optimisation can be employed which provide better coverage of the performance surface. However, the computational overhead associated with concurrent search is significant.

\section{Case study description}

The case study considers a two dimensional (2D) NWT, whereby the NWT is one cell thick and symmetry planes are imposed on the front and back faces of the domain. The 2D NWT is used to allow timely investigation of the optimal experiment design, before moving to the much computationally slower $3 \mathrm{D}$ NWT for real WEC geometries.

The NWT is implemented using OpenFOAM, as detailed in [14]. The present case considers a 50m deep tank with walls $100 \mathrm{~m}$ from the device and with wave creation/absorption implemented via the waves2FOAM package [15] utilising two $90 \mathrm{~m}$ long relaxation zones situated $10 \mathrm{~m}$ either side of the device. A 2D circular device geometry is simulated, which relates to the cross-section of a horizontally aligned cylinder of infinite length. The radius of the cylinder is $1 \mathrm{~m}$ and it's density is set to half of the water so that it rests $50 \%$ submerged. Fig.5 shows a view of the mesh around the cylinder. The cylinder's motion is constrained to heave in all experiments.

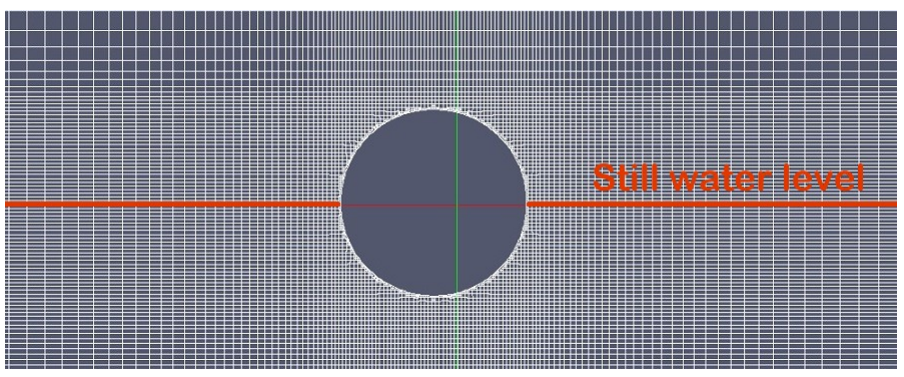

FIGURE 5. Side view of the 2D geometry and the mesh used in the CFD simulation

\section{NWT experiments}

NWT experiments are divided into two categories: Preliminary experiments, which are used to inform the construction of the signals used for system identification, and the identification tests themselves.

\subsection{Preliminary Experiments}

Two simple preliminary experiments can be used to determine the frequency bandwidth of the device's natural and forced responses. Both preliminary experiments involve a very low total amount of kinetic energy in the device and the NWT fluid, which results in very fast CFD simulations. This allows the preliminary experiments to be quickly run before the identification experiments to inform the design of the identification signals' frequency content.

The device's natural response is determined via a free decay experiment, whereby the device is initially displaced from its equilibrium position and allowed to freely oscillate back to rest. The upper trace in Fig.6, shows a free decay experiment performed on the case study set-up and the lower trace displays the Fourier transform of the free decay and gives an indication of the device resonance and bandwidth (state dynamics).
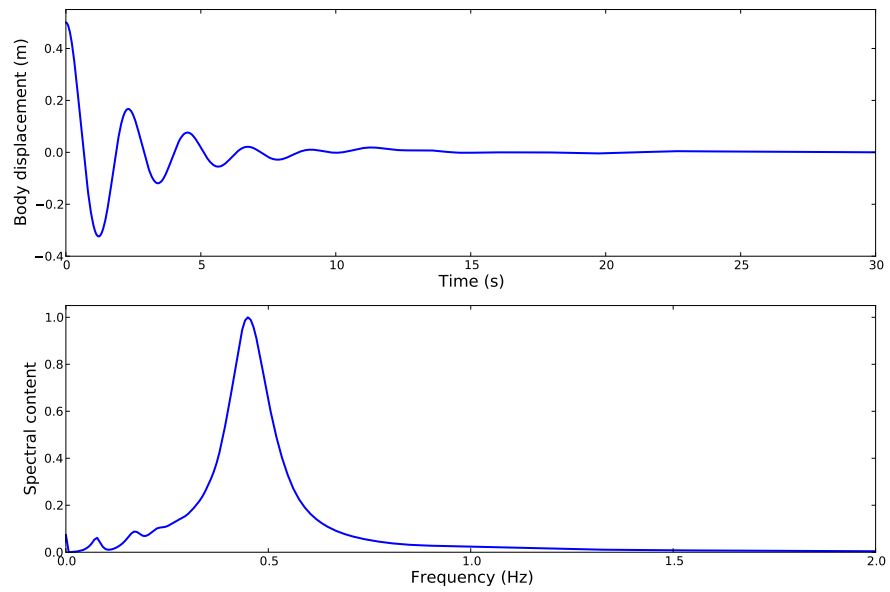

FIGURE 6. Device free response in time and frequency domains

The device's forced response is determined via a small amplitude chirp signal experiment, whereby a chirp signal is applied as a direct force on the device. Unlike the chirp signal used for the later identification experiments, which must cover a desired amplitude range, the chirp signal in the preliminary experiments is of very low amplitude allowing fast simulation times. The small amplitude chirp signal experiment therefore gives a frequency response identification of the total forced dynamics around the equilibrium position. This response is shown 


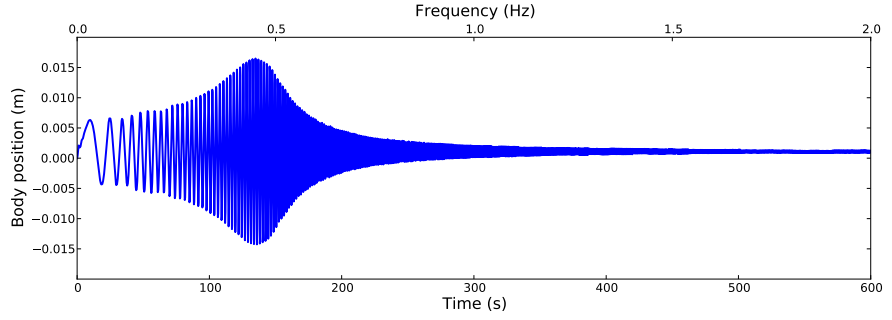

FIGURE 7. Preliminary frequency response determination using a small amplitude chirp signal

\subsection{Identification experiments}

The identification experiments consider two types of tests, whereby the device motion is either driven by wave excitation or by a direct force applied to the device. Two different signal types are investigated for the direct force tests, a chirp and a RARP input, while a multisine signal is used for the wave excitation tests. Note that a RARP signal cannot be used to synthesise a real sea variation. The details of the identification tests are as follows:

Wave excitation test The device motion is excited by input waves generated in the NWT. The input wave signal is a 600s multisine signal consisting of 100 equally spaced frequencies from $0.005-0.995 \mathrm{~Hz}$ with randomly assigned phases and amplitudes determined from a JONSWAP spectrum with a peak period of $8 \mathrm{~s}$, significant wave height $\left(H_{S}\right)$ of $0.6 \mathrm{~m}$ and a peakiness $(\gamma)$ value of 2 .

Chirp input A 600s chirp signal, sweeping the frequency range up to $1.5 \mathrm{~Hz}$ and with a maximum amplitude of $1 \mathrm{kN} / \mathrm{m}$, was input as a direct force on the device.

RARP input A 600s RARP signal, with maximum allowable switching period of $0.67 \mathrm{~s}$ and maximum allowable amplitude of $1 \mathrm{kN} / \mathrm{m}$, was input as a direct force on the device.

\section{System identification}

In this section, the specific nonlinear modelling approach is documented, together with the details of the particular force and wave excitation signals used, along with the parametric form of the various ANN models identified.

\subsection{Modelling approach}

As a nonlinear modelling tool, ANNs are selected, since they provide a generic nonlinear function approximation capability [16] with scalable complexity. Since the main focus of

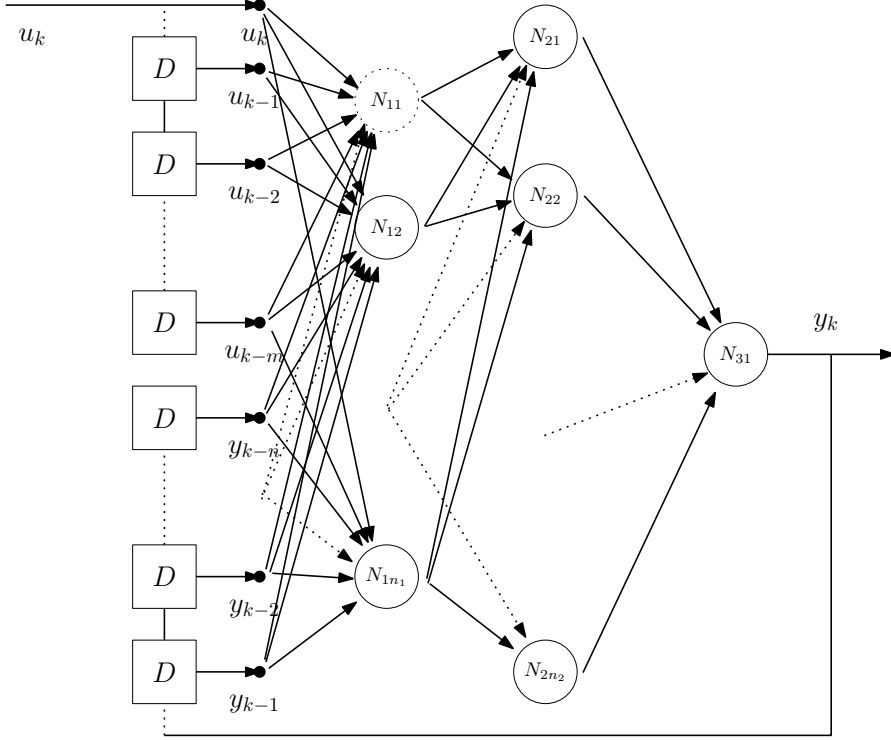

FIGURE 8. Dynamic neural network structure

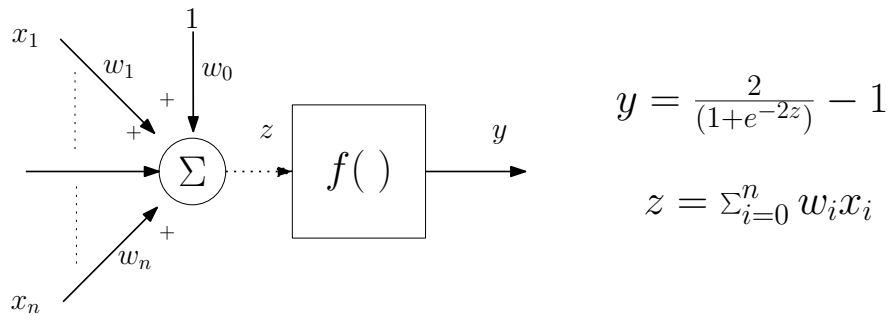

FIGURE 9. Structure of an individual neuron

the paper is on numerical wave tank identification signals, the specific nonlinear parameterisation of the identified models is of lesser importance. Dynamics are incorporated into the nonlinear ANN models by the use of tapped delay lines (TDLs) at the network input layer, as shown in Fig.8, where each $D$-block denotes a single delay element. There are a number of structural aspects of the model to be specified, as part of the modelling procedure:

(a) The number of layers in the network,

(b) The number of neurons in each layer,

(c) The type of neuron to use,

(d) The number of delayed input values to use, $m$, and

(e) The number of delayed output values, $n$.

While one layer of nonlinear neurons can give arbitrary approximation capability, two nonlinear layers usually requires a lower total neuron count, for a given level of approximation accuracy. We choose perceptron-type neurons, where the tansigmoidal basis functions (see Fig.9) provide global support and give a smooth interpolation between training data points, resulting in a multi-layer perceptron (MLP). The overall nonlinear 


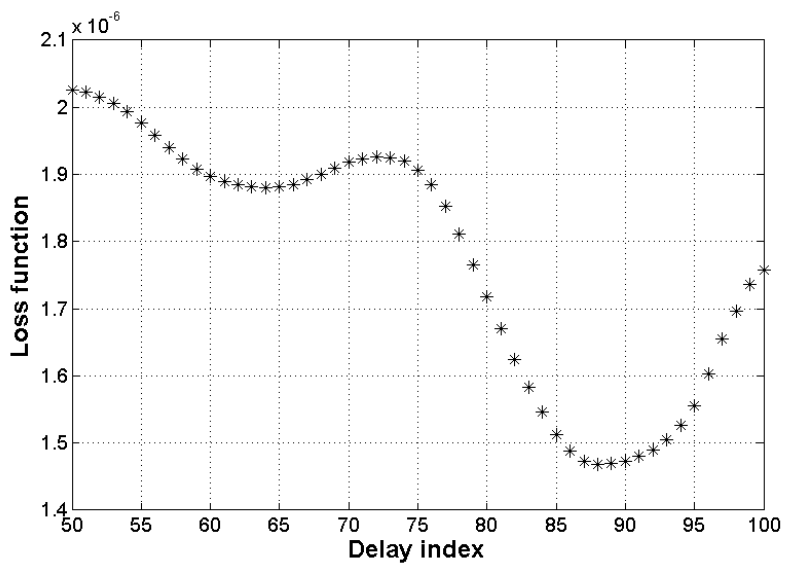

FIGURE 10. Determination of model anti-causality

complexity is determined by $n_{1}$ and $n_{2}$, the number of neurons in layers 1 and 2, respectively. $n_{1}$ and $n_{2}$ are determined by growing the network incrementally, until a good balance is achieved between approximation of the training data and generalisation capability.

The dynamical order of the system $(n$ and $m$ ) is determined using linear modelling tools. A range of linear models are built for various values of $n$ and $m$ and the loss function (a measure of the modelling error) on validation data monitored. The least complex model (smallest $n$ and $m$ ), which gives adequate modelling capability, is chosen i.e. a parsimonious model.

For a model relating free surface elevation to device displacement, an additional step must be taken, since this relationship is, in general, noncausal. To this end, the input signal $u$ is advanced a number of steps, $n k_{\max }$ to ensure that the response is causal and the optimal delay from this offset (for a linear model) determined. For example, for a sampling period of $0.1 \mathrm{~s}$, a value of $n k_{\max }=100$ is used, equivalent to an advance of $10 \mathrm{~s}$. Then, using suitable values for $n$ and $m$, the number of delay steps $n k$, which minimises the loss function on validation data, is determined, as shown in Fig.10. This results in a net required advance of $12(=100-88)$ steps to achieve the required level of noncausality.

Finally, having determined the structure of the model in Fig.8, the parameters (i.e. the weights and biases) of the network are determined using an optimisation algorithm. A scaled conjugate gradient (backpropagation) search algorithm [17] is employed for parameter determination, using a variety (100 instances) of initial conditions to counter the difficulty of local minima in the search surface.

\subsection{Wave excitation inputs}

When the excitation is provided by incoming waves, a noncausal model (as described in Section 6.1) is used and Fig.11

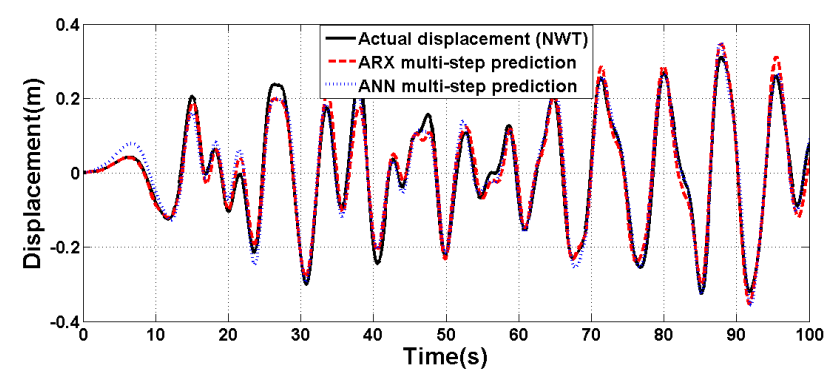

FIGURE 11. Model performance on training data for wave excitation

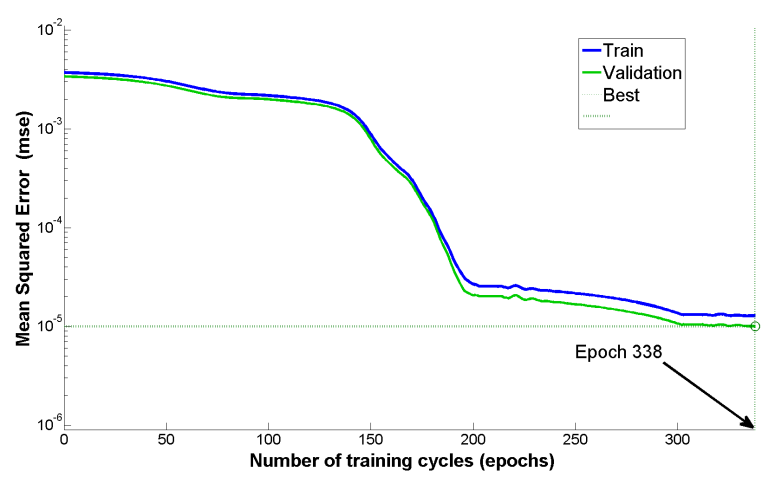

FIGURE 12. Example training record for ANN

shows the performance of an ANN model $\left(n_{1}=3, n_{2}=5, n=8\right.$, $m=2$ ) on the training data. By way of example, the training record for the ANN in Fig.12 is shown; note that the training is halted as soon as the error on validation data starts to rise (after 338 training cycles), termed early stopping. Summary results, using the normalised root mean-squared error (NRMSE) as an error metric, are shown in Table 1. The main observation is that neither modelling approach is perfect, but the linear model has particular difficulty in following peaks in the displacement, suggesting that some nonlinear phenomena are present in the motion due to this wave excitation signal. To show the comparability of wave and direct force excitations, in terms of amplitude of body motion, Fig.13 shows the relative displacement (distinct from body position) of the device with respect to water surface. This will be seen to be commensurate with the relative displacement for the direct force tests, where no external waves are present.

\subsection{Direct force application}

Force can be directly applied to the device as a PTO signal and can take the form of multisines, chirp signals or RARP signals, as outlined in Section 3.2. Fig.14 shows the performance of an ANN model $\left(n_{1}=3, n_{2}=5, n=4, m=2\right)$ on the training data for a large-amplitude chirp input, while the performance of an ANN model (also $n_{1}=3, n_{2}=5, n=4, m=2$ ) on the training 


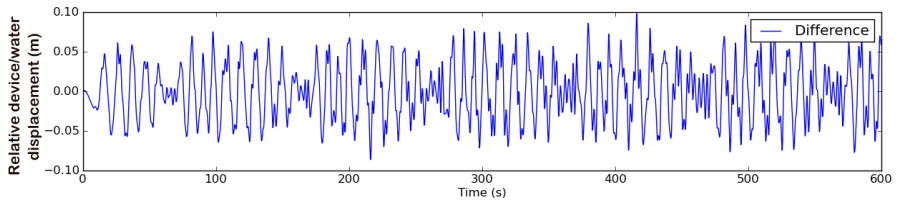

FIGURE 13. Relative displacement of device with respect to water surface for wave excitation

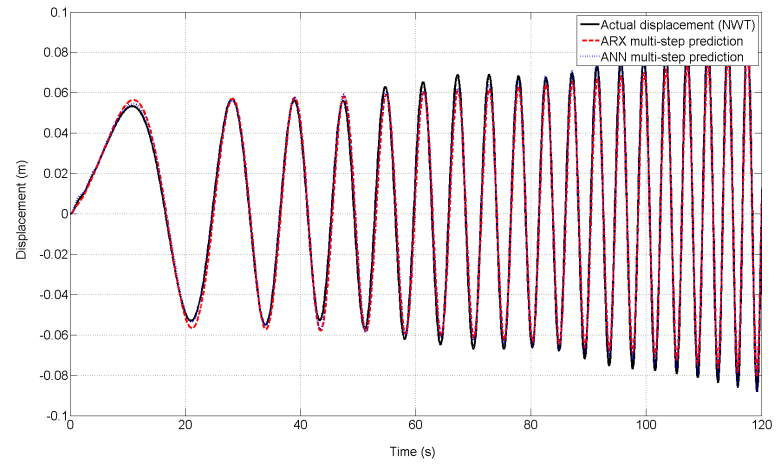

FIGURE 14. Model performance on training data for direct excitation using a chirp signal

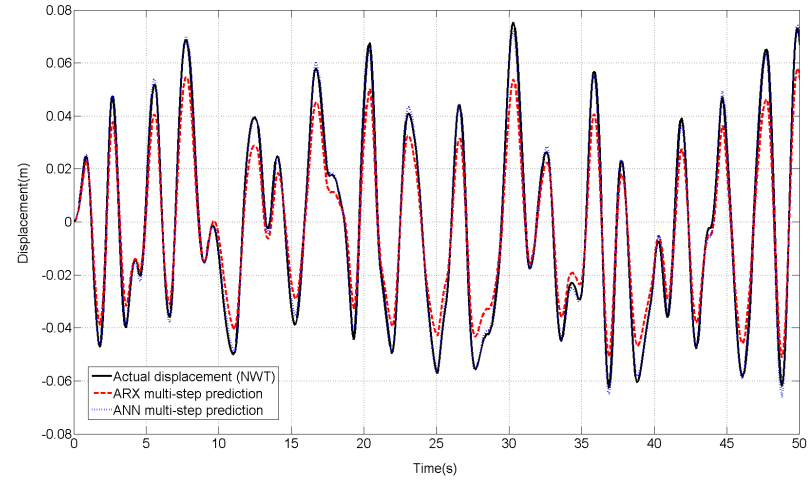

FIGURE 15. Model performance on training data for direct excitation using a RARP signal

data for the RARP signal is shown in Fig.15. From Table 1, it can be seen that all models perform reasonably well on both training and validation data, though with significant improvement for the ANN models over their linear ARX counterparts.

\section{Model validation}

Here, the performance of the identified models on previously unseen data is evaluated, with summary results in Table 1.

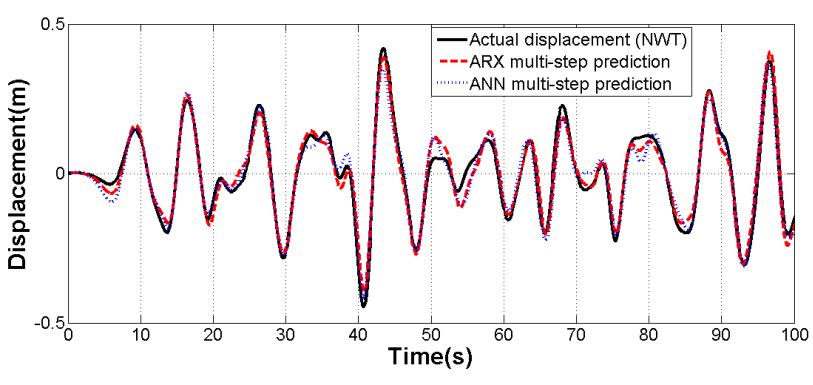

FIGURE 16. Model performance on validation data for wave excitation

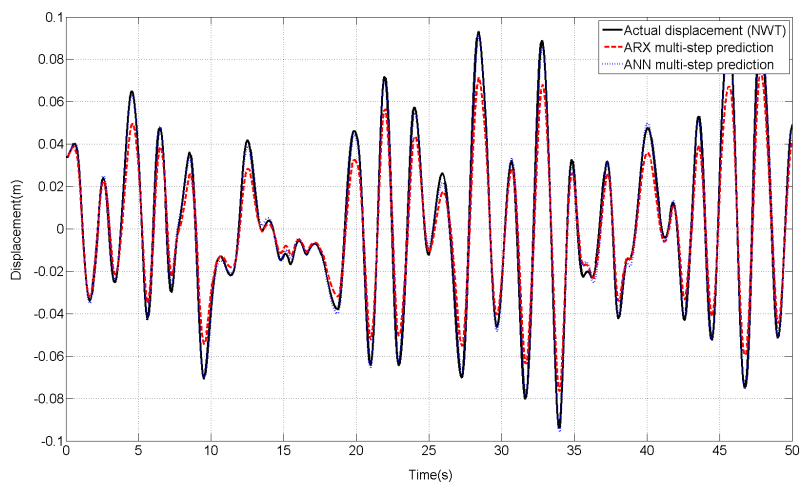

FIGURE 17. Model performance on validation data for direct excitation using a RARP signal

\subsection{Wave excitation inputs}

Fig.16 shows the performance of an ANN model, identified on wave excitation training data, for a different wave excitation. Given the relatively small deterioration in NRMSE for the validation case, it can be reasonably argued that both ARX and ANN models generalise well. The slightly greater deterioration in validation performance for the ANN model is not unsurprising, though a surprising feature is that the ANN model is outperformed by the linear ARX model. This may suggest that there is a need for further work on the selection of a parsimonious structure for the ANN, or that a good global minimum of the performance surface has not been reached in training.

\subsection{Direct force application}

The performance of the ANN model, trained on the training data for the RARP signal, using a different RARP input, is shown in Fig.17. The relatively small difference in the performance metric for the ANN model on the RARP training and validation data suggests that the nonlinear model generalises well, indicating a parsimonious structure for the network (number of inputs and number of layers/neurons). 


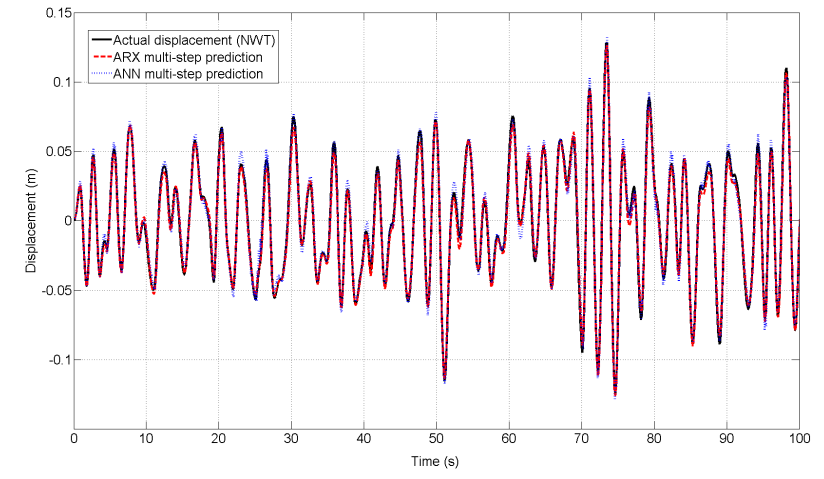

FIGURE 18. Chirp model performance on cross-validation data for direct excitation using a RARP signal

\subsection{Cross-validation}

Here, we show the performance of a model trained on a certain excitation type, but validated on a different excitation type i.e. cross validation. For the wave input case, only one type of signal can be synthesised, so no cross-validation tests can be performed at this input point. However, for the direct force application, Fig.18 shows the performance of the ARX and ANN models identified on the chirp data, but fed with a RARP input. Similarly, Fig.19 shows the performance of the ARX and ANN models identified on the RARP data, but fed with a chirp input. Table 2 summarises the relative merits of various identification signals, in a cross-validation test. It is interesting that the performance of the chirp ANN model on the RARP signal input is quite good; not as good as the ANN RARP model (100\% worse), but performance is quite acceptable. Similarly, the ANN RARP model performs well for a chirp input, with a NRMSE of around $400 \%$ compared to the ANN chirp model. While it is tempting to conclude that the chirp signal therefore provides a better general excitation, it is safer to conclude that both chirp and RARP signals provide excitation signals for identification that generalise well to other input signal forms. The simplicity of the chirp signal is also a strength and it's coverage of the amplitude space is also evident from Fig.4.

\section{Discussion}

Table 1 describes the performance of the identified models on training and validation data, using the NRMSE as a metric. For comparison, results obtained using a linear (ARX) model, similar to that in (2), are also included. Note that there are no validation results for the chirp signal excitation, since it's not possible to produce a significantly different validation excitation for a chirp. While it difficult to compare the absolute NRMSE values for models fed with different excitation inputs, the comparative values allow some conclusions to be drawn.

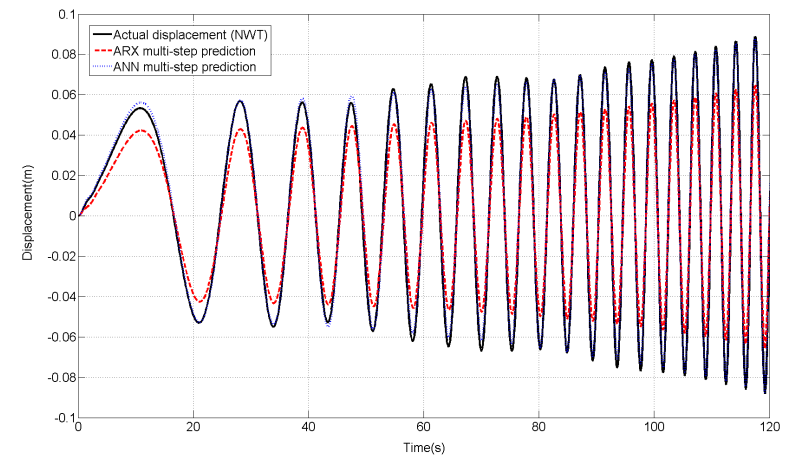

FIGURE 19. RARP model performance on cross-validation data for direct excitation using a chirp signal

\begin{tabular}{|c|c|c|c|}
\hline Excitation & Error metric & Training & Validation \\
\hline \multirow{2}{*}{ Wave } & ARX NRMSE & 0.1761 & 0.1827 \\
& ANN NRMSE & 0.1847 & 0.2109 \\
\hline \multirow{2}{*}{ Chirp force } & ARX NRMSE & 0.0702 & - \\
& ANN NRMSE & 0.0441 & - \\
\hline \multirow{2}{*}{ RARP force } & ARX NRMSE & 0.2364 & 0.2340 \\
& ANN NRMSE & 0.0536 & 0.0545 \\
\hline
\end{tabular}

TABLE 1. Model performance for training and validation

It is clear that the ANN models outperform the ARX models in the vast majority of cases, so we can broadly conclude that nonlinear models are required to model the NWT data for the device under test. It is, of course, significant that the device under test has a nonlinear restoring force due to the heterogeneous cross-sectional area. Those cases where the ARX model better the ANN model may well correspond to situation where a good (local or global) minimum of the ANN performance surface was difficult to find, which is one drawback of using ANN models.

In general, the validation, including cross-validation, errors for the various models are commensurate with those for training, indicating that the models have a good ability to generalise. This suggests that parsimonious models have been achieved, ensured by choosing modest values for $n_{1}, n_{2}, n$ and $m$, and stopping ANN training as soon as the validation error starts to rise.

The cross-validation results assess the value of various (direct force) excitation signals for the NWT. From the modest set of cross-validation results presented, both chirp and RARP excitation signals appear to result in models which generalise well to the other type of excitation, so broad conclusion can be reached that both RARP and chirp are good excitation signals for the identification of nonlinear black-box models. 


\begin{tabular}{|c|c|c|}
\hline Excitation & Error metric & Cross-validation \\
\hline Chirp force & ARX NRMSE & 0.2369 \\
(RARP model) & ANN NRMSE & 0.1873 \\
\hline RARP force & ARX NRMSE & 0.0827 \\
(chirp model) & ANN NRMSE & 0.1224 \\
\hline
\end{tabular}

TABLE 2. Model performance for cross validation

\section{Conclusions}

Setting up the NWT to perform identification tests is nontrivial; there are many issues surrounding (for example) the appropriate choice of mesh and the tank length, as a good compromise between fidelity of response and computational burden. In addition, a variety of excitation signals may be applied at the force input stage, while significant limitations exist for variations in the free surface elevation. In particular, no sharp edges can be synthesised in the wave signal, so RARP-type signals cannot be used. However, chirp and multisine signals, which have a basis in system identification [13], can be employed as wave input signals, which have direct analogies with (instantaneous) monochromatic seas and polychomatic seas, respectively.

The optimisation of NWT identification experiments is an iterative process, where preliminary tests should be performed in order to correctly set up the NWT. In addition, identification signals should be optimised, so that maximum model identifiability capacity for a given computational load is achieved. From the data presented, and the cross-validation tests in particular, it appears that both RARP-type signals and chirp signals are effective identification signals at the direct force input point, while wave excitation is constrained to time series which can be modelled by real sea model spectra e.g. Jonswap.

\section{Acknowledgement}

This project is funded by Enterprise Ireland and is co-funded by the Irish Government and the European Union under Irelands EU Structural Funds Programme 2007- 2013 under grant $\mathrm{EI} / \mathrm{CF} / 2011 / 1320$.

\section{REFERENCES}

[1] Nievergelt, Y., 2000. "A tutorial history of least squares with applications to astronomy and geodesy". Journal of Computational and Applied Mathematics, 121(12), pp. 37 -72 .

[2] Ljung, L., 2010. "Perspectives on system identification". Annual Reviews in Control, 34(1), pp. 1-12.

[3] Ljung, L., 1999. System Identification (2Nd Ed.): Theory for the User. Prentice Hall PTR, Upper Saddle River, NJ, USA.

[4] Prony, R., 1795. "Essai experimental et analytique: Sur les lois de la dilatabilite des fluides elastiques et sur celles de la force expensiver de la vapeur del'eau et de la vapeur de l'alkool, a differentes temperatures". Paris J. l'Ecola Polytechnique, 1, pp. 24-76.

[5] Perez, T., and Fossen, T. I., 2009. "A Matlab Toolbox for Parametric Identification of Radiation-Force Models of Ships and Offshore Structures". Modeling, Identification and Control, 30(1), pp. 1-15.

[6] Bhinder, M. A., Babarit, A., Gentaz, L., and Ferrant, P., 2011. "Assessment of viscous damping via 3d-cfd modelling of a floating wave energy device". In Proceedings of the 9th European Wave and Tidal Energy Conference, Southampton, UK.

[7] Davidson, J., Giorgi, S., and Ringwood, J., 2013. "Linear parametric hydrodynamic models based on numerical wave tank experiments". In Proc. European Wave and Tidal Energy Conf. (EWTEC), Aalborg.

[8] Astrom, K. J., and Wittenmark, B., 1994. Adaptive Control, 2nd ed. Addison-Wesley Longman Publishing Co., Inc., Boston, MA, USA.

[9] Goodwin, G., and Payne, R., 1977. Dynamic system identification : experiment design and data analysis. Academic Press, New York, USA.

[10] Tanizawa, K., 2000. "The state of the art on numerical wave tank". In Proc. 4th Osaka Colloquium on Seakeeping Performance of Ships, pp. 95-114.

[11] Currie, I., 1974. Fundamental Mechanics of Fluids. McGraw-Hill.

[12] Rao, G., and Unbehauen, H., 2006. "Identification of continuous-time systems". Proc. IEE-Control Theory Appl., 153, pp. 185-220.

[13] Pintelon, R., and Schoukens, J., 2012. System Identification - A Frequency Domain Approach (2nd Edition). Wiley, Hoboken, NJ.

[14] Davidson, J., Giorgi, S., and Ringwood, J., 2014. "Numerical wave tank identification of nonlinear discrete-time hydrodynamic models". In Proc. 1st Int. Conf. on Renewable Energies Offshore (Renew 2014), Lisbon.

[15] Jacobsen, N. G., Fuhrman, D. R., and Fredsøe, J. "A wave generation toolbox for the open-source CFD library: Openfoam R',

[16] Cybenko, G., 1989. "Approximation by superpositions of a sigmoidal function". Mathematics of control, signals and systems, 2(4), pp. 303-314.

[17] Møller, M. F., 1993. "A scaled conjugate gradient algorithm for fast supervised learning". Neural Networks, 6(4), pp. 525-533. 